\title{
SOLID DISSOLUTION IN FALLING FILMS OF NON-NEWTONIAN LIQUIDS*
}

\author{
R. A. MASHELKAR AND V. V. GHAVAN \\ Department of Chemical Engineering, University of Salford, \\ Lancashire, England
}

\begin{abstract}
The problem of solid dissolution in films of non-Newtonian pseudoplastic liquids falling over flat, cylindrical, spherical and conical surfaces is studied theoretically. The resulting Sturm-Liouville problem is solved by series expansion on a digital computer. Numerical results for the eigenvalues, eigenfunctions, expansion coefficients and average expansion coefficients are reported for power-law liquids. Semi-quantitative considerations are presented for considering the influence of fluid-stretching in the case of viscoelastic liquids.
\end{abstract}

\section{Introduction}

Although a considerable interest has been shown in recent years in the study of momentum and heat transfer characteristics of non-Newtonian fluids, relatively little work has been done so far as the problems of mass transfer are concerned. In numerous industrial situations, such as diffusion-controlled polymerisation reactions, several biological and physiological processes as well as in many industrial waste disposal systems, a knowledge of mass transfer in non-Newtonian media may be extremely important.

One of the important factors influencing the rates of mass transfer is the molecular diffusion coefficient of the solute in the solvent. There is a good deal of information in the literature on the diffusion coefficients of solutes in a variety of non-Newtonian media such as polymer solutions, suspensions etc. (see e.g. 1, 2, 9, 13). It has been conclusively proved that the conventional formulae for computing the diffusion coefficients in Newtonian media give erroneous results when used for non-Newtonian media. It is found that in spite of the enormous differences in the viscosities of the solvents and the polymer solutions, the order of magnitude of the diffusion coefficients in the polymer solutions is practically the same as that in the solvent.

Since most non-Newtonian liquids are highly viscous in character, the conventional static techniques for the determination of diffusion coefficients may be unsuitable. For instance, Hansford and Litt ${ }^{11)}$ attempted to measure the diffusivity of benzoic acid in

* Received on July 22, 1972

Dr. R. A. Mashelkar, Dept. of Chem. Eng., Univ. of Salford, Salford M 54 W T, Lancashire, England polymer solutions by using diaphragm cell technique, but the results were not reliable. Further, the nonNewtonian behaviour of the material will be exhibited only under flow conditions. Hence it is important to devise techniques where measurements of mass transfer rates in flowing non-Newtonian liquids under welldefined flow conditions can be made. This will enable us to calculate the value of the diffusion coefficient in the non-zero shear rate region. This will also enable us to examine the effect on the diffusion coefficient, if any, of the changes in the molecular configurations which occur in a flowing non-Newtonian liquid.

One such technique is the study of the rate of dissolution of solids in flowing films of non-Newtonian liquids. Usually flat and inclined slabs of the solids to be dissolved have been used for such a purpose2). However the possibility of using other shapes has not been investigated so far.

In this work, we have studied theoretically the problem of solid dissolution in non-Newtonian liquids which are flowing over flat surfaces and surfaces having some degree of symmetry. In particular, we have chosen two shapes, viz. spherical and conical. These might offer some advantage in terms of reducing the end effects which exist when working with flat and cylindrical surfaces. The problem has been solved numerically and the resulting solutions have been compared with the solutions obtainable under limiting conditions.

\section{Background}

There is very little work reported in the literature where either the theoretical or the experimental aspects of solid-liquid mass transfer in the non-zero shear rate region have been studied. The main contributions in 
this area will be first briefly reviewed and critically analysed.

Clough, Read, Metzner and Behn ${ }^{5)}$ were the first to study the problem of solid dissolution in flowing nonNewtonian liquids. Soluble cast pipes of Benzoic acid and $\beta$-naphthol were used and the rate of mass transfer from the solid wall into non-Newtonian liquids flowing under fully developed laminar-flow conditions was studied. The theoretical solution used was the mass transfer equivalent of the limiting heat transfer solution given by Lévéque ${ }^{15)}$, now popularly known as the Lévéque approximation. The mass transfer data were used to find out the diffusivities of the solutes in flowing non-Newtonian liquids. It is obvious that such a setup, although ingenious, will suffer from the experimental difficulties of casting solute pipes as well as having lengthy test pieces and recirculation pumps.

Astarita ${ }^{2)}$ studied the rates of mass transfer from a solid inclined plate made of the solute material into thin films of non-Newtonian solutions flowing over it. The mechanism of wall slip at the solid surface was postulated and the mass transfer rates were theoretically calculated based on an arbitrary velocity distribution of the type $v=a_{m} x^{m}$, where $0 \leq m \leq 1$. The solution obtained was restricted particularly to cases where the axial distances were small or flow rates were rather large, so that the Lévéque approximation could be assumed to be valid.

Hansford and Litt ${ }^{11}$ studied the solid dissolution rates from a soluble discs rotating in non-Newtonian media in the laminar regime. The convective diffusion equation was solved by using the velocity distribution around the disc obtained by Mitschka and Ulbrecht ${ }^{20)}$. The latter solution was valid for discs rotating in inelastic power-law liquids under laminar boundary layer flow conditions. There was, however, a wide discrepancy between the theoretical results and the experimental data. This was attributed to the fact that the flow field around the disc was significantly different from that assumed in the theoretical derivation. The theoretical velocity distribution, which was valid only for inelastic liquids, could not describe the experimentally observed secondary flow effects which arise out of the interaction of the elastic and inertial forces. The rates of mass transfer were used to calculate the diffusivities of the solutes in non-Newtonian media. But because of the drawbacks pointed above, the results could not be relied upon.

We have considered here the problem of solid dissolution in non-Newtonian films which are flowing over flat or cylindrical surfaces. The theoretical solution is obtained numerically over a wide range of variables and is not, hence, restricted in the way the Lévéque approximation is. The solution is later extended to bodies which have a certain degree of symmetry. In particular, we have considered the dissolution rates on a solid sphere where there is a stretching and a contracting of the film, and on a solid cone where there is a continuous contracting of the film. The influence of these factors on the mass transfer rates are considered in detail. It is anticipated that the theoretical results could suitably be used for studying solid dissolution rates under well-defined flow conditions, and hence these will be of help in obtaining reliable data on diffusion coefficients.

\section{Previous Work on Solid Dissolution in Newto- nian Fluids}

The problem of solid dissolution in Newtonian fluids flowing over flat as well as spherical and conical surfaces has been studied to some extent in the past.

When the penetration of the solute in the flowing film is very small, the concentration boundary layer near the solid wall with a constant velocity gradient is usually considered, and the solution obtained is in accordance with the classical Lévéque approximation. Kramers and Kreyger ${ }^{14)}$ used this solution for studying the mass transfer rates from dissolving inclined plates made out of the solute. Unfortunately this theoretical solution is applicable only in a narrow range of variables, i.e. for short axial lengths or very high flow rates. In heat transfer terminology, one might say that the Graetz number has to be sufficiently high. An extension of this solution to non-Newtonian fluids is fairly elementary because the problem essentially reduces to calculating the velocity gradient at the wall for the non-Newtonian fluid under consideration.

When the Lévéque approximation is inadequate, the presence of the entire velocity profile in the film must be taken into account. A detailed numerical solution for the resulting Sturm-Liouville problem for the Newtonian liquid has been made by several workers (e.g. 4, 25, 26). The corresponding problem for non-Newtonian fluids, however, has not been yet solved.

An interesting situation exists when a laminar film of a fluid flows over spherical or conical surfaces. In this case, there is some stretching and contracting of the film as the film flows over the surface. This stretching of the film results in a distortion of the concentration gradient as the film flows from the top to the bottom. For instance, in the case of a sphere, there is a gradual increase in the concentration gradients in the upper half and a decrease in the lower half. The overall influence of this phenomenon on the rate of gas absorption in Newtonian films has been studied ${ }^{18)}$. However, there is no information in the literature in which the influence of these conditions on solid dissolution rates in non-Newtonian fluids has been mentioned.

The fluid mechanics problem of the flow of liquids on spherical and conical surfaces has not been solved so far. However, all the existing analyses $\left.{ }^{7}, 21,23,28\right)$ have made use of the assumptions of Lynn, Straatmeier and Kramers ${ }^{16)}$ to describe the film hydrodynamics. These authors assumed that the film thickness of the liquid flowing at any latitude on the sphere is the same as it would be for the same flow rate per unit length on an inclined plane making the same angle with the vertical. 
There is plenty of experimental evidence in the literature to suggest that these assumptions are valid.

Davidson, Cullen, Harrison and Roberts ${ }^{8)}$ measured the dynamic holdup of a string of spheres and compared it with the one calculated, with the above assumption. There was excellent agreement between the two values. Further, Davidson and Cullen ${ }^{7)}$ used the above assumptions to calculate theoretically the rates of gas absorption, and calculated therefrom the diffusivities of the gases. There was good agreement between the values thus obtained and those reported in the literature. Another proof has been obtained by Wild and Potter ${ }^{28)}$, who have directly measured the film thickness on a single sphere using a projected-shadow technique and found that the assumptions are justified.

Although the problem of solid dissolution in spherical films has not been solved so far, the technique of Davidson and Cullen ${ }^{7)}$ for solving the problem of gas absorption may be employed in this case as well. Davidson and Cullen simplified the diffusion equation by using the streamlines as co-ordinate framework. The latter approach has been used by Goldstein ${ }^{10)}$ for solving the heat conduction problems in non-rotational motion. Although the problem of the flow of a film on a sphere is a rotational one, the same simplifications could be made by assuming a parabolic form of the velocity distribution for a Newtonian liquid at every radial section.

Olbrich and Wild ${ }^{21)}$ have extended the approach suggested by Davidson and Cullen for other geometrical systems which have a degree of symmetry to such an extent that only two space variables are sufficient to specify the system.

\section{Theory}

\section{1) Solid dissolution in flat non-Newtonian films}

Consider the laminar film of a solvent falling over a flat plate made of a sparingly soluble material. Under steady-state isothermal conditions, the relevant convective diffusion equation is given by

$$
v_{z} \frac{\partial c}{\partial z}=D \frac{\partial^{2} c}{\partial x^{2}}
$$

It has been assumed that diffusive transport in the axial direction $z$ is negligible as compared to convective transfer.

For a power-law fluid whose flow behaviour is defined by

$$
\tau_{x z}=K\left(\frac{-d v_{z}}{d x}\right)^{1 / N}
$$

the velocity distribution will be given by

$$
v_{z}=v_{\max }\left(1-(x / \delta)^{N+1}\right)
$$

The results for the interfacial velocity $v_{\max }$ and the film thickness $\delta$ will be given by

$$
v_{\max }=\frac{1}{N+1}(d g / k)^{N} \delta^{N+1}
$$

$$
\delta=\left((N+2) \frac{Q}{W(d g / k)^{N}}\right)^{\frac{1}{N+2}}
$$

The equivalent results for $v_{\max }$ and $\delta$ for a film falling over a vertical cylinder of radius $R$ can be simply obtained by substituting $W=2 \pi R$.

Eq.(1) can be made dimensionless by defining dimensionless variables $\rho=x / \delta, \quad \theta=\left(C^{*}-C\right) /\left(C^{*}-C_{0}\right)$ and $\xi=z D / v_{\max } \delta^{2}$. Eq.(1) will then be transformed as

$$
\left(1-\rho^{N+1}\right) \frac{\partial \theta}{\partial \xi}=\frac{\partial^{2} \theta}{\partial \rho^{2}}
$$

The boundary conditions for the solution of Eq.(6) may be written as

$$
\begin{array}{ll}
\xi=0, & \theta=1 \\
\rho=0, & \frac{\partial \theta}{\partial \rho}=0
\end{array}
$$

and

$$
\rho=1, \quad \theta=0
$$

Eq. (6) with B. C. (7), (8) and (9) can be solved by separating the variables and letting

$$
\theta=f(\rho, \xi)=\phi_{i}(\rho) \phi_{i}(\xi)
$$

Substituting Eq.(10) in Eq.(6) and rearranging to separate the axial and radial part of the solution, we get

$$
\frac{1}{\phi_{i}} \frac{d \phi_{i}}{d \xi}=\frac{1}{\left(1-\rho^{N+1}\right) \phi_{i}} \frac{d^{2} \phi_{i}}{d \rho^{2}}=-\lambda_{i}
$$

where $\lambda_{i}$ are the eigenvalues. The axial part of the solution using B. C. (7) is given by

$$
\phi_{i}(\xi)=e^{-\lambda_{i} \xi}
$$

and the radial part $\phi(\rho)$ is given by

$$
\frac{d^{2} \phi_{i}}{d \rho^{2}}+\phi_{i} \lambda_{i}\left(1-\rho^{N+1}\right)=0
$$

Eq.(13) because of its B. C. is of the Sturm-Liouville type. The concentration profiles are of the form

$$
\theta(\rho, \xi)=\sum_{i=1}^{\infty}(-1)^{i+1} B_{i} \phi_{i}(\rho) \phi_{i}(\xi)
$$

where $B_{i}$ are the expansion coefficients and $\phi_{i}(\rho)$ are the eigenfunctions. The latter are obtained in the series form

$$
\phi_{i}(\rho)=\sum_{j=0}^{\infty} a_{i, j} \rho^{j}
$$

Using B. C. (8), we get $a_{i, 1}=0$. Further, $a_{i, 0}$ will be assumed to be equal to one. The remaining coefficients can be obtained from the recursion formula

$$
a_{i, j}=\lambda_{i}\left[\frac{a_{i, j-N-3}-a_{i, j-2}}{j(j-1)}\right]
$$

An additional condition is obtained by using the B. C. (9), when one obtains

$$
\sum_{j=0}^{\infty} a_{i, j}=0
$$

The expansion coefficients $B_{i}$ can be evaluated by using the orthogonality condition as 


$$
B_{i}=\frac{\int_{0}^{1} \phi_{i}\left(1-\rho^{N+1}\right) d \rho}{\int_{0}^{1} \phi_{i}{ }^{2}\left(1-\rho^{N+1}\right) d \rho}
$$

The average concentration of the dissolved solute at any dimensionless axial distance $\xi$ can be calculated by defining an average dimensionless concentration $\theta_{\text {avg }}(\xi)$ as

$$
\theta_{a v g}(\xi)=\frac{C^{*}-\bar{C}}{C^{*}-C_{0}}=\frac{\int_{0}^{1} \theta v_{z}(\rho) d \rho}{\int_{0}^{1} v_{z}(\rho) d \rho}=\sum_{i=1}^{\infty} A_{i} e^{-\lambda_{i} \xi}
$$

where $A_{i}$ and $\lambda_{i}$ are the 'average' expansion coefficients and the eigenvalues, respectively.

Another useful parameter $G$, which is the fractional degree of saturation may be defined as

$$
G=\frac{\bar{C}-C_{0}}{C^{*}-C_{0}}=1-\theta_{a v g}(\xi)
$$

We will also express the results in terms of the Sherwood number $\left(S h=k_{L} \delta / D\right)$ as

$$
S h=\frac{1-\theta_{\text {avg }}(\xi)}{\xi\left(v_{\max } / v_{\text {avg }}\right)}
$$

\section{2) Lévéque approximation}

Kramers and Kreyger ${ }^{14)}$ have solved the problem of solid dissolution in a film of a Newtonian fluid falling over a flat surface by assuming the presence of a. constant velocity gradient in the thin concentration boundary layer near the wall. The result may be expressed as

$$
k_{L}=0.808\left(\frac{\stackrel{\circ}{\gamma}_{w} D^{2}}{z}\right)^{1 / 3}
$$

For the case of a power-law fluid, the velocity gradient at the wall, $\stackrel{\circ}{\gamma}_{w}$ is given by $v_{\max }(N+1) / \delta$. Hence expressions for Sherwood number, $S h$, and the degree of saturation, $G$, may be obtained as

$$
\begin{gathered}
S h=0.808\left(\xi \frac{v_{\max }}{v_{\text {avg }}}\right)^{-1 / 3}(N+2)^{1 / 3} \\
G=0.808\left(\frac{N+2}{(N+1)^{2 / 3}}\right) \xi^{2 / 3}
\end{gathered}
$$

The function of $N$ in the bracket in Eq.(24) incorporates the effect of pseudoplasticity on the mass transfer rate. As indicated earlier this solution will be valid only under the conditions when the axial lengths are very small or the flow velocities are rather large, or, in other words, this will be valid only for rather small values of $\xi$. A comparison of Eq. (24) with the results of the numerical solution will be made later.

\section{3) Solid dissolution in films falling over spher- ical and conical surfaces}

Fig. 1 shows the picture of a thin film flowing over a cylindrical, spherical and conical surface. The changes in dimension of the film as it flows from the top to the bottom are defined as shown in the figure. As shown by Olbrich and Wild ${ }^{21)}$, the flow system here could be considered to be symmetrical to an extent such

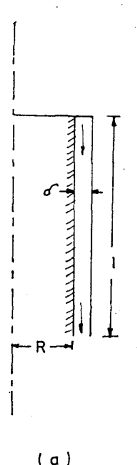

Circular cylinder

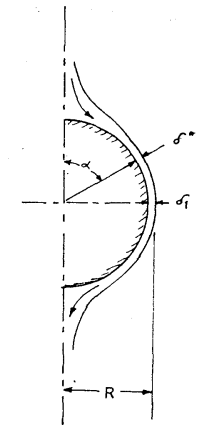

(b)

Sphere

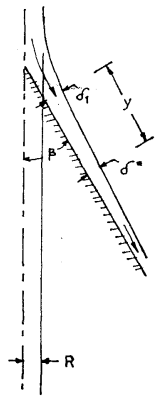

(c)

Cone
Fig. 1 Various flow geometries used in this work

that only two variables are sufficient to specify it, say $s$ (in the direction of flow) and $x$ (in the direction transverse to the flow). The relevant convective diffusion equation may then be written as

$$
v_{s} \frac{\partial c}{\partial s}=D \frac{\partial^{2} c}{\partial x^{2}}
$$

The velocity $v_{s}$ will be given by a distribution

$$
v_{s}=v^{*}\left(1-\left(\frac{x}{\delta^{*}}\right)^{N+1}\right)
$$

Note that the surface velocity $v^{*}$ and the film thickness $\delta^{*}$ are not constant, but are functions of the variables. Thus for the case of a sphere the variable $s$ will correspond to the angle $\alpha$, whereas in the case of a cone $s$ will correspond to the distance $y$ along the cone. Since $v^{*}$ and $\delta^{*}$ are functions of $s$ only, another variable $\chi$ may be defined such that

$$
\frac{d \chi}{d s}=\frac{D}{v^{*} \delta^{* 2}}
$$

Substituting Eq.(26) and Eq.(27) in Eq.(25), we obtain in the dimensionless form,

$$
\left(1-\left(\rho^{*}\right)^{N+1}\right) \frac{\partial \theta}{\partial \chi}=\frac{\partial^{2} \theta}{\partial \rho^{* 2}}
$$

The B.C. for the solution of Eq.(28) may now be written as

$$
\begin{aligned}
& \chi=0, \quad \theta=1 \\
& \rho^{*}=0, \quad \frac{\partial \theta}{\partial \rho^{*}}=0 \\
& \rho^{*}=1, \quad \theta=0
\end{aligned}
$$

Since the B. C. (29), (30) and (31) are identical with the B.C. (7), (8) and (9), the numerical solution obtained for Eq.(6) will be valid in this case as well. The variable $\chi$ will have to be evaluated by using Eq. (27), when we have

$$
\chi=\int_{0}^{s} \frac{D}{v^{*} \delta^{* 2}} d s
$$

The evaluation of $\chi$ in the particular case of a sphere and a cone are outlined below.

Assuming that the film thickness $\delta^{*}$ of the liquid flowing at any latitude on the sphere is the same as 
Table 1 The value of the integral in Eq.(37) as a function of the pseudoplasticity

$N$

\begin{tabular}{rc}
$N$ & Integral \\
\hline 1 & 1.6826 \\
2 & 1.6524 \\
3 & 1.6351 \\
4 & 1.6238 \\
5 & 1.6160 \\
6 & 1.6101 \\
7 & 1.6056 \\
8 & 1.6020 \\
9 & 1.5991 \\
10 & 1.5967 \\
$\infty$ & 1.5708 \\
\hline
\end{tabular}

Table 2 Comparison of $\lambda_{i}, B_{i}$ and $A_{i}$-Newtonian fluids This work

\begin{tabular}{rccrrrr}
\multicolumn{1}{c}{$\lambda_{i}$} & $B_{i}$ & $A_{i}$ & & \multicolumn{1}{c}{$\lambda_{i}$} & $B_{i}$ & $A_{i}$ \\
\hline 2.8278 & 1.2008 & 0.9104 & & 2.8278 & 1.2008 & 0.9104 \\
32.2147 & 0.2992 & 0.0531 & & 32.1470 & 0.2991 & 0.0531 \\
93.4749 & 0.1608 & 0.0153 & & 93.4750 & 0.1608 & 0.0153 \\
185.7320 & 0.1049 & 0.0066 & & 186.8000 & 0.1074 & 0.0068 \\
\hline
\end{tabular}

Table 3 The eigenvalues $\left(\lambda_{i}\right)$

\begin{tabular}{rrrrr}
$N=1$ & $N=2$ & $N=3$ & $N=4$ & $N=\infty$ \\
\hline 2.8278 & 2.6466 & 2.5711 & 2.5317 & 2.4674 \\
32.2147 & 28.1320 & 26.1506 & 24.9995 & 22.2066 \\
93.4749 & 81.5672 & 75.6685 & 72.1724 & 61.6851 \\
185.7320 & 161.9550 & 150.1481 & 143.2100 & 119.7950 \\
\hline
\end{tabular}

Table 4 The expansion coefficients $\left(B_{i}\right)$

\begin{tabular}{lllll}
$N=1$ & $N=2$ & $N=3$ & $N=4$ & $N=\infty$ \\
\hline 1.2008 & 1.2171 & 1.2286 & 1.2369 & 1.2732 \\
0.2992 & 0.3235 & 0.3408 & 0.3537 & 0.4244 \\
0.1608 & 0.1745 & 0.1836 & 0.1910 & 0.2546 \\
0.1049 & 0.1144 & 0.1208 & 0.1261 & 0.1817 \\
\hline
\end{tabular}

Table 5 The average expansion coefficient $\left(A_{i}\right)$

\begin{tabular}{lllll}
$N=1$ & $N=2$ & $N=3$ & $N=4$ & $N=\infty$ \\
\hline 0.9104 & 0.9004 & 0.8922 & 0.8855 & 0.8106 \\
0.0531 & 0.0589 & 0.0637 & 0.0676 & 0.0900 \\
0.0153 & 0.0170 & 0.0184 & 0.0197 & 0.0324 \\
0.0066 & 0.0074 & 0.0080 & 0.0087 & 0.0166 \\
\hline
\end{tabular}

would be obtained for the same flow rate per unit length on an inclined plane making the same angle with the vertical, we get

$$
\delta^{*}=\delta_{1} \sin \alpha^{-\frac{N+1}{N+2}}
$$

Where $\delta_{1}$ is the film thickness at the equator $(\alpha=\pi / 2)$ and is given by

$$
\delta_{1}=\left[(N+2) \frac{Q}{2 \pi R(d g / k)^{N}}\right]^{\frac{1}{N+2}}
$$

The maximum velocity $v^{*}$ at the surface of the film can be easily calculated as

$$
v^{*}=V \sin \alpha^{-\frac{1}{N+2}}
$$

where

$$
V=(N+1)(d g / k)^{N} \delta_{1}{ }^{N+1}
$$

Substituting Eq.(33) and Eq.(35) in Eq.(32), we obtain

$$
\chi=\frac{D R}{V \delta_{1}{ }^{2}} \int_{0}^{\pi}(\sin \alpha)^{\left(\frac{2 N+3}{N+2}\right)} d \alpha
$$

The expression under the integral sign represents the effect of stretching and contracting of the film as the liquid flows over the surface of the sphere. For a Newtonian liquid $(N=1)$, the expressions for $\chi$ have been obtained by Davidson and Cullen ${ }^{7)}$ and Olbrich and Wild ${ }^{21)}$ and they are exactly the same as in Eq.(37).

The corresponding expression for the cone may be obtained similarly. Thus, with reference to Fig. 1 and with the assumptions mentioned earlier, the expressions for $\delta^{*}, \delta_{1}$ and $v^{*}$ are given by

$$
\begin{gathered}
\delta^{*}=\delta_{1}\left[\frac{1}{1+\sin \beta(y / R)}\right]^{\frac{1}{N+2}} \\
\delta_{1}=\left[(N+2) \frac{Q}{2 \pi R\left(\frac{d g \cos \beta}{k}\right)^{N}}\right]^{\frac{1}{N+2}} \\
V^{*}=V\left[\frac{1}{1+\sin \beta(y / R)}\right]^{\frac{N+1}{N+2}}
\end{gathered}
$$

where

$$
V=\left(\frac{1}{N+1}\right) \cdot\left(\frac{d g \cos \beta}{k}\right)^{N} \delta_{1}{ }^{N+1}
$$

Combining Eq.(32), (38) and (39), we obtain

$$
\chi=\frac{D R}{V \delta_{1}^{2}} \int_{0}^{l}(1+\sin \beta(y / R))^{\frac{3+N}{2+N}} d y
$$

The integral in this case represents the effect of the contracting of the film. The expression for $\delta^{*}$ in Eq. (38) for a Newtonian fluid $(N=1)$ is the same as that reported by Bird, Stewart and Lightfoot ${ }^{3)}$. Further, the expression for $\chi$ (Eq.(42)) for a Newtonian fluid is identical to that obtained by Olbrich and Wild ${ }^{16)}$.

The Integral in Eq.(37) had to be computed numerically. Table 1 gives the values of this integral as a function of the reciprocal flow behaviour index. The integral in Eq.(42) can, however, be obtained analytically, and hence $\chi$ will be given by

$$
\begin{aligned}
\chi= & \frac{D R}{V \delta_{1}^{2}} \frac{1}{\sin \beta}\left(\frac{N+2}{2 N+5}\right) \\
& \times\left[\left(1+\sin \beta\left(\frac{y}{R}\right)\right)^{\frac{2 N+5}{N+2}}-1\right]
\end{aligned}
$$

\section{Results and Conclusion}

All the computations were performed by using an ICL-KDF9 digital computer. The eigenvalues were determined by solving the polynomial obtained from Eqs. (15) and (16) using the Bairstow method ${ }^{24)}$. The eigenfunctions were computed from Eq.(15) by considering the first 300 terms. The infinite series for the expansion coefficient $A_{i}$ were obtained and these values were also calculated in the same fashion.

It was thought desirable to check the accuracy of the results of the present work with those available in the 
literature. Brown ${ }^{4)}$ has solved the same problem for a Newtonian fluid $(N=1)$ and has extended the series solution to ten terms. The present solution is restricted to four terms.

The first four eigenvalues, $\left(\lambda_{i}\right)$, expansion coefficients $\left(B_{i}\right)$ and the average expansion coefficients $\left(A_{i}\right)$ obtained in this work are compared with those obtained by Brown ${ }^{4)}$ in Table 2. Excellent agreement is found between the two sets of values. Brown has also compared his values with those obtained by some other workers (e.g. 25), 26)), and his paper may be referred to for a further comparison. The results of the numerical work are shown in Tables 3 to $\mathbf{5}$. Table 3 gives the eigenvalues $\lambda_{i}$ obtained for various values of the power-law index and Table 4 gives the expansion coefficients $B_{i}$. The average expansion coefficients $A_{i}$ are reported in Table 5.

The overall influence of the non-Newtonian flow behaviour of the material on the rate of solid dissolution is seen by calculating the Sherwood number, $S h$, as a function $\left(\xi\left(V_{\max } / V_{a v g}\right)\right)$ for various levels of pseudoplasticity. The result of increasing pseudoplasticity is found to be to increase the rate of solid dissolution (see Fig. 2). This observation may be compared with the situation existing in the case of gas absorption in falling films of non-Newtonian fluids ${ }^{12}$. In the latter case increasing pseudoplasticity tends to decrease the rate of gas absorption. It is thus evident that it is not possible to decide upon the influence of the non-Newtonian behaviour of the material on the rates of heat and mass transfer without considering the individual problem from a fundamental viewpoint.

Lévéque solution is a good approximation for very small values of $\xi$. However, even for small $\xi$, Lévéque solution will be in greater and greater error as the pseudoplasticity of the material increases, and will completely fail as $N \rightarrow \infty$. For a Newtonian fluid $(N=$ 1), the error in using Lévéque approximation for $\xi=$ 0.1 and 0.3 is $0.75 \%$ and $12.2 \%$, respectively. However, for a pseudoplastic liquid with $N=4$, the error in using Lévéque approximation for $\xi=0.1$ and 0.3 is $16.4 \%$ and $26.9 \%$ respectively. This implies that in the experimental determination of diffusion coefficients Lévéque approximation should be used for shorter mass transfer sections $(\xi \ll 0.1)$, but for larger mass transfer sections $(\xi \gg 0.1)$ and for highly pseudoplastic liquids $(N>3)$, the results of this work should be used.

The net result of pseudoplasticity with stretching and contracting of the film can be examined by considering the case of a sphere. For a Newtonian fluid $(N=1)$, the value of $\chi$ in expression (37) will be given by $\xi=$ $D(1.68 R) / V \delta_{1}{ }^{2}$ (see Table 1). Comparing this expression with the case of a circular cylinder of radius $R$, it could be easily deduced that if the length of the circular cylinder is $1.68 R$, then the rates of mass transfer at equal flow rates will be the same in both the cases. Thus $1.68 R$ may be considered to be an "equivalent length" of the cylinder for obtaining equal rates of mass transfer. The value of the integral in Eq.(37) thus indicates

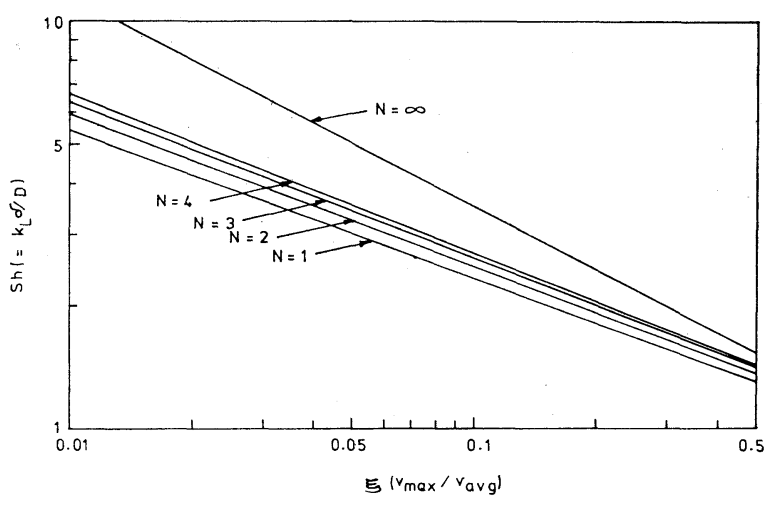

Fig. 2 Effect of pseudoplasticity on the mass transfer rates

the net effect of stretching and contracting. It is clearly seen from Table 1 that the effect of increasing pseudoplasticity is to decrease the "equivalent length" of the cylinder.

The analysis presented in this work has not taken into account the end effects and rippling. However, the geometries analysed in this work have some advantages. Thus for instance in the case of a sphere the end effects may be reduced substantially because they may be confined to the supporting rod at the top of the sphere ${ }^{6)}$. Further, the vertical extent of the liquid surface will be short enough to avoid rippling without the addition of surface active agents. Also, the studies of Oliver and Atherinos ${ }^{22)}$ clearly indicate that the rates of solid dissolution can be adequately predicted by the theory of smooth laminar film even if rippling is present. Conditions for using these geometries for non-Newtonian liquids are likely to be further favourable. This is because of the high consistency of non-Newtonian liquids, which will bring the Reynolds number nearer to zero and reduce the problem of accounting for the presence of developing velocity profile still further.

The analysis presented in this work refers to inelastic liquids only. The same analysis is applicable for viscoelastic liquids which are flowing over flat and cylindrical surfaces, since under such steady laminar shearing conditions the velocity profiles will be unaffected by the elastic properties of the material. In the case of viscoelastic fluids flowing over spheres and cones the situation is likely to be different.

As viscoelastic fluids flow down on such geometries, the fluids are subjected to changing deformation rates. Several workers ${ }^{17-19)}$ have shown that in materials capable of elastic and viscous responses only the former are operative if the deformation rates are changing sufficiently rapidly. In other words, under such conditions viscoelastic fluids show a "solidlike" response. In the case of the sphere, for instance, one intuitively feels that such effects are likely to be present in the topmost and the bottommost region, since the flows are rapidly decelerating and accelerating, respectively, in these regions. We now attempt a more rigorous semi-quantitative verification of the possibility of such 
fluid behaviour.

We will use the approach of previous workers $\left.{ }^{17}, 18\right)$ to explore this problem by using the "limiting stretch rate" hypothesis. The convected Maxwell model ${ }^{27)}$ in which the stresses $\tau^{i j}$ are related to the deformation rates $d^{i j}$ by a viscosity function $\mu$ and a relaxation time fuction $\theta^{\prime}$ appears to portray the most important properties of the material in a simple manner, and may be written as

$$
\tau^{i j}=2 \mu d^{i j}-\theta^{\prime} \frac{\delta \tau^{i j}}{\delta t}
$$

where the symbol, $\delta / \delta t$ represents the Oldroyd contravariant convected derivative. The stress developed in the steady stretching of a flat sheet of viscoelastic material described by means of the above model, with a single relaxation time $\theta^{\prime}$, is given by (17) as

$$
\Delta \tau=\frac{4 \mu \Gamma_{l}}{1-\left(2 \theta \Gamma_{l}\right)^{2}}
$$

Here $\Gamma_{l}$ denotes the local stretch rate and $\Delta \tau$ is the excess normal stress traction along the streamline. As the term $2 \Gamma \theta_{l} \rightarrow 1$, the stresses become larger and thus if the fluid is to remain continuous the viscoelastic fluid will possess a finite maximum stretch rate. If no anomalous effects are to be observed, then we must satisfy the condition

$$
2 \theta^{\prime} \Gamma_{l} \ll 1
$$

The order of magnitude of the local stretch rate in the case of a fluid flowing over a sphere can be estimated by approximating the velocity distribution by the Newtonian distribution $(N=1)$. Thus considering Eq.(26), the local stretch rate or the rate of change of local velocity along a streamline may be calculated as

$$
\Gamma_{l} \simeq \frac{1}{r}\left(\frac{d v_{s}}{d \alpha}\right)
$$

It is conceivable that the maximum stretch rates will occur at the surface. Since the film thickness $\delta^{*}$ at any position on the sphere will be much smaller than the radius of the sphere $R$, we approximate $R+\delta^{*} \simeq R$ and obtain the magnitude of the stretch rate at the surface, $\Gamma_{s}$ as

$$
\left.\Gamma_{s} \simeq \frac{d v_{s}}{r d \alpha}\right|_{r=R+\delta^{*}} \simeq \frac{V}{3 R} \frac{\cos \alpha}{(\sin \alpha)^{4 / 3}}
$$

Combining Eqs. (46) and (48) with $\Gamma_{s}=\Gamma_{\iota}$ we obtain the inequality

$$
\frac{2 \theta^{\prime} V}{3 R} \frac{\cos \alpha}{(\sin \alpha)^{4 / 3}} \ll 1
$$

Substituting practical values of $V \simeq 10 \mathrm{~cm} / \mathrm{sec}, R \simeq 1$ $\mathrm{cm}, \theta^{\prime} \simeq 10^{-2} \sec$ (for reasonably dilute polymer solutions), we find that excluding the region of approximately 7 degrees from the top and the bottom the inequality will be satisfied. Thus in the majority of the region on the sphere, no anomalous effects will be observed. It should be emphasized here that this analysis overemphasizes the influence of stretching in the region far removed from the surface of the sphere. Fur- ther, the analysis is an order of magnitude estimation and is based on the assumption of a well-developed flow region. It is quite likely that elasticity will have a significant influence on the flow pattern at the stagnation point, and consequently on the well-developed flow region as well. In view of these complexities the present analysis should be regarded only as an approximation limited of validity.

\section{Conclusion}

The problem of solid dissolution in films of nonNewtonian pseudoplastic liquids is studied theoretically. The flow geometries are conveniently chosen so that they may be used for experimental determination of molecular diffusivities of solutes in pseudoplastic liquids in non-zero shear rate region over rather a wide range of variables. Numerical results are presented for power-law liquids. The influence of fluid-stretching in the case of viscoelastic liquids is considered semiquantitatively by using a limiting stretch rate hypothesis.

\section{Nomenclature}

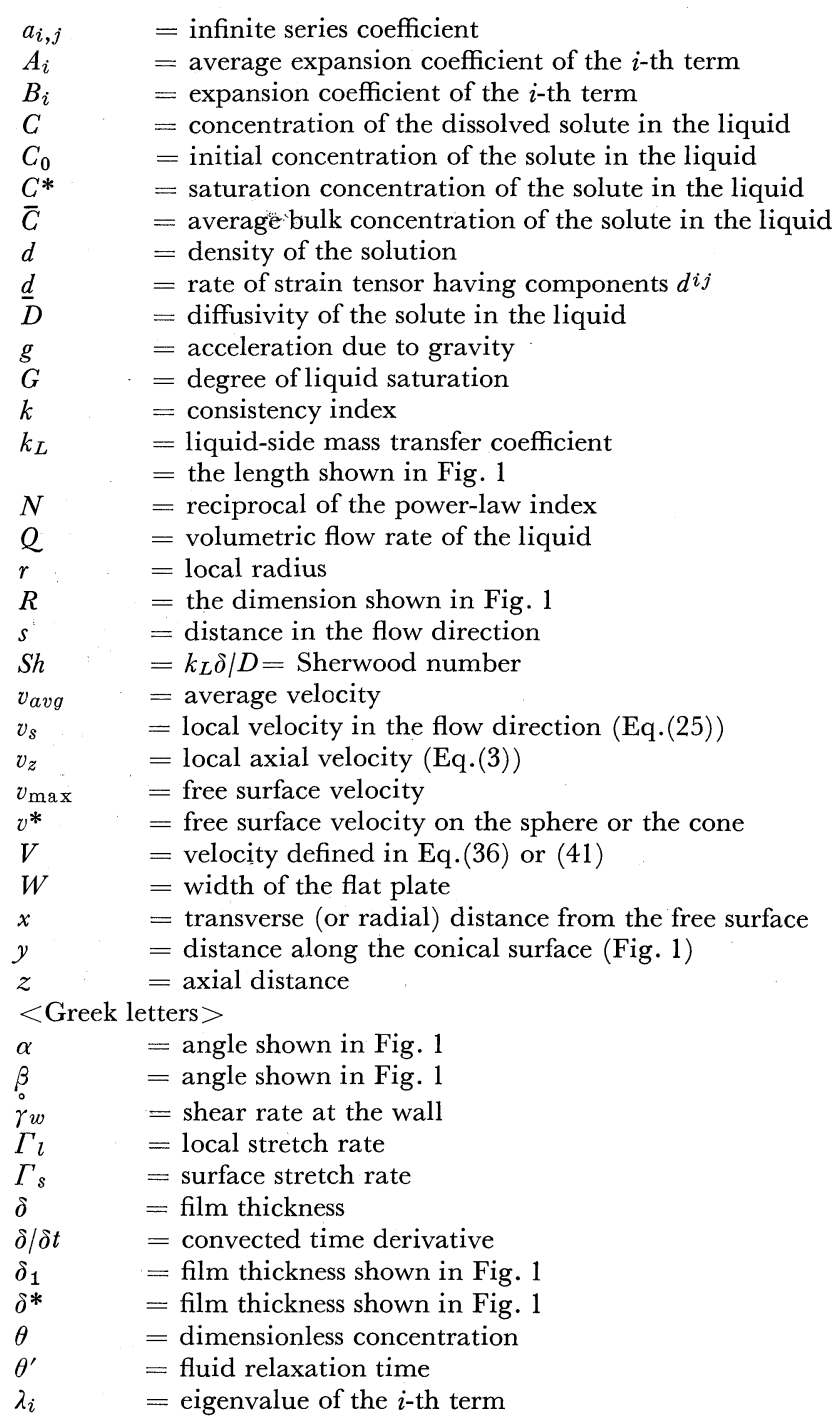




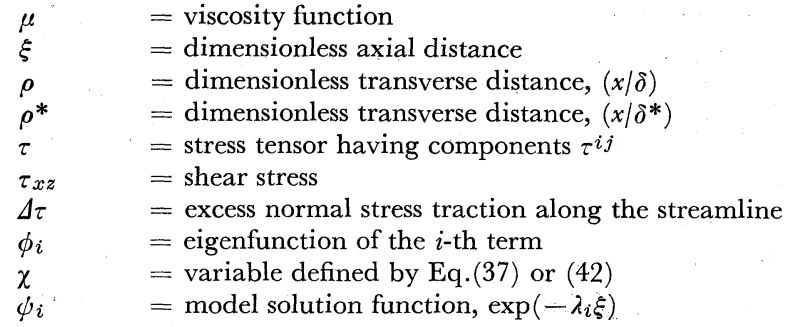

\section{Literature Gited}

1) Astarita, G.: I.E.C. Fund., 4, 236 (1965)

2) Astarita, G.: I.E.C. Fund., 5, 14 (1966)

3) Bird, R. B., W. E. Stewart and E. W. Lightfoot: "Transport Phenomena", New York: John Wiley and Sons Inc. (1960)

4) Brown, G. M.: A. I. Ch. E. J., 6, 179 (1960)

5) Glough, S. B., A. E. Read, A. B. Metzner and V. C. Behn: $A$. I. Ch. E. J., 8, 346 (1962)

6) Danckwerts, P. V.: “Gas-Liquid Reactions", New York, McGraw-Hill (1970)

7) Davidson, J. F. and E. J. Cullen: Trans. Inst. Chem. Eng. (London), 53, 51 (1957)

8) Davidson, J. F., E. J. Cullen, D. Harrison and D. Roberts: Trans. Inst. Chem. Eng. (London), 37, 122 (1959)

9) Dim, A., G. R. Gardner, A. B. Ponter and T. Wood: J. Chem. Eng. Japan, 4, 92 (1971)

10) Goldstein, S.: "Modern Developments in Fluid Dynamics", Vol. II, Oxford University Press (1938)
11) Hansford, G. S. and M. Litt: Chem. Eng. Sci., 23, 849 (1968)

12) Hirose, T. and M. B. Moo-Young: Can. J. Chem. Eng., 47, 265 (1969)

13) Hoshino, S. and K. Sato: Kagaku Kögaku, 31, 961 (1967)

14) Kramers, H. and P. J. Kreyger: Chem. Eng. Sci., 6, 42 (1956)

15) Lévéque, J.: Ann. Mines, 13, 201, 305, 381 (1928)

16) Lynn, S., J. R. Straatmeier and H. Kramers: Chem. Eng. Sci., 4, $63 \cdot(1955)$

17) Metzner, A. B.: A. I. Ch. E. J., 13, 317 (1967)

18) Metzner, A. B. and G. Astarita: A. I. Ch. E. J., 13, 550 (1967)

19) Metzner, A. B., J. L. White and M. M. Denn: Chem. Eng. Progr., 62, (12), 81 (1966)

20) Mitschka, P. and J. Ulbrecht: Appl. Scient. Res., A15, 345 (1965)

21) Olbrich, W. E. and J. D. Wild: Chem. Eng. Sci., 24, 25 (1969)

22) Oliver, D. E. and T. E. Atherinos: Chem. Eng. Sci., 23, 525 (1968)

23) Ratcliff, G. A. and J. G. Holdcroft: Chem. Eng. Sci., 15, 100 (1961)

24) Scarborough, J. B.: "Numerical Mathematical Analysis", Baltimore, John Hopkins Press (1966)

25) Schenk, J. and J. M. Dumove: Appl. Scient. Res., A4, 39 (1953)

26) Sellars, J. R., M. Tribus and J. S. Klein: Trans. A. S. M. E., 78, 441 (1956)

27) White, J. L. and A. B. Metzner: J. Appl. Polymer Sci., 7, 1867 (1963)

28) Wild, J. D. and O. E. Potter: I. Chem. E. Symp. Ser., No. 28 (Inst. Chem. Engers, London), 30, (1968)

29) Zandi, I. and C. D. Turner: Chem. Eng. Sci., 25, 517 (1970)

\title{
MASS TRANSFER IN THE CONTINUOUS PHASE AROUND A SINGLE DROP*
}

\author{
Koretsune UEYAMA, Kanehiro OGAWA** \\ AND JuN-IGHI HATANAKA \\ Department of Chemical Engineering, University of Osaka \\ Prefecture, Sakai, Japan
}

\begin{abstract}
A simple model is proposed for mass transfer in a continuous phase around a single liquid drop. The model is based upon the assumption that the ratio of the average Sherwood number for a drop to that for its equivalent solid sphere is equal to the ratio of the local values at their front stagnation points. The available data for some binary systems are reasonably well correlated with the model, and it is theoretically more consistent than the analogical models proposed by Griffith, and Shirotsuka and Hirata which are based upon the boundary-layer analysis of the flow over a flat interface moving with a constant velocity.
\end{abstract}

\section{Introduction}

Mass transfer in a liquid medium around a single

\footnotetext{
* Received on August 5, 1972

Presented at the Okayama Meeting of the Soc. of Chem. Engrs., Japan, Okayama, July 10, 1972.

** Kobe Steel Co., Ltd., Kobe

于591 堺市百舌鳥梅町4-804

大阪府立大学工学部化学工学科 畑中淳一
}

drop is one of the fundamental problems of liquidliquid extraction. Many investigations have been presented experimentally or theoretically for the problem. But the hydrodynamic analysis of the system of a drop and surrounding fluid is very difficult, even in the case of steady translating motion of the drop, because of the moderate Reynolds numbers in cases of practical interests.

Several authors have suggested analytical methods $^{2,10,22)}$ based upon the boundary-layer flow calculat- 